\title{
New electrochemical approach for the measurement of oxidative DNA damage: Voltammetric determination of 8- oxoguanine at screen-printed graphite electrodes
}

4

\author{
Elena Bernalte ${ }^{a, b}$, Michael Carroll ${ }^{a}$ and Craig E. Banks ${ }^{a *}$
} Extremadura, Avda. de Elvas s/n, 06006, Badajoz, Spain.
a: Faculty of Science and Engineering, Manchester Metropolitan University, Chester Street, Manchester M1 5GD, UK.

${ }^{b}$ : Departamento de Química Analítica e IACYS, Facultad de Ciencias, Universidad de 
30 Simplification and miniaturisation of analytical methods for the direct detection of DNA damage

31 is a challenging area of research and screen-printed electrodes are a promising alternative

32 approach to analytically / electroanalytically monitor the species involved. In this work we

33 demonstrate that screen-printed graphite macroelectrodes (SPEs) provide useful

34 electrochemical signatures to study the behaviour of 8-oxoguanine (8-oxoGua), which is the

35 most frequent and important marker of oxidative DNA damage and it is widely considered as a

36 biomarker, via differential pulse voltammetry (DPV). Under the optimum experimental

37 conditions, the proposed electrochemical sensing protocol towards 8-oxoGua using SPEs is

38 demonstrated to be possible over the concentration range of 0.1 to $12 \mu \mathrm{M}$. The response of the

39 SPEs is superior over routinely utilised glassy carbon electrodes in terms of sensitivity with a limit

40 of detection $(3 \sigma)$ found to correspond to $0.33 \mu \mathrm{M}$. Reproducibility and repeatability of the

41 proposed methodology at low and high concentrations were also demonstrated. Quantification

42 of 8-oxoGua in the presence of other nucleobases and different compounds of interest which

43 are present in biological fluids was successfully accomplished. Furthermore, proof-of-concept

44 demonstrating the potential use of the developed SPE based methodology for the detection of

45 8-oxoGua in real complex samples as demonstrated in simulated biological samples (human

46 semen).

47 Keywords: Screen-printed electrodes; 8-oxoguanine; DNA oxidation; Voltammetry 


\section{Introduction}

DNA is incessantly being damaged. In addition to known external factors, such as chemical agents, UV light or ionising radiation, a human cell must repair around 10,000 DNA lesions per cell, per day, that are due to multiple endogenous sources.[1] Oxidative stress is the most common and persistent cause of DNA destruction and it is generated through multiple processes involving production of oxygen and nitrogen species such as nitric oxide and superoxides.[2] In particular, these reactive oxygen species (ROS) have the capacity to attack cells and react with a variety of cellular components[1], including nucleotides of DNA, causing particularly hazardous damage for cells that can lead to genomic instability involved in various pathological conditions as well as mutation and cancer.[3, 4]

Guanine is the primary target of ROS in DNA[5] because it has the lowest redox potential of the four nucleobases.[6] 8-oxoguanine (8-oxoGua hereinafter) which contains an extra oxygen atom on guanine derived from the oxidation in the C8 position, is the most frequent and important oxidative DNA alteration in all aged cell types[7] and it is widely considered as a biomarker of oxidative DNA damage.[5] These modifications on the nitrogen bases abolish their ability to hydrogen-bond with the complementary bases and thus mismatch insertion or replication blockage, which can result in gene mutations.[2] In this particular case, 8-oxoGua mispairs with adenine during DNA replication that causes GC to TA transversion mutation.[3]

The most common biological samples that are examined in general for oxidative stress markers are blood, serum, urine, nasal and lung lavages, biopsied human tissue, and animal or human tissues or cells, as well as sperm, breast milk, placenta, and saliva are matrixes of interest.[8] Consequently, substantial analytical difficulties can be found in the analysis of this compound in those complex biological samples. As described in the literature, determination of 8-oxoGua has been performed by several methods such as high performance liquid chromatography with electrochemical detection (HPLC-ECD), gas chromatography coupled to mass spectrometry (GC-MS), liquid chromatography with mass spectrometry (LC-MS/MS) and enzyme linked immunosorbent assay (ELISA).[9] One of the most popular methods for the direct analysis of the released 8-oxoGua is HPLC-ECD [10-14] even though possible interference of the biological matrix and time required for the analysis of large number of samples are significant drawbacks[9]. GC-MS is also a suitable analytical technique for 8-oxoGua quantification mainly because of its sensitivity[9], but overestimation compared to HPLC-ECD, attributed to the artificial oxidation of guanine base during typical derivatisation reaction used prior GC-MS, is the main reported disadvantage.[9-11] On the other hand, specificity, sensitivity, capability to 
quantify multiple analytes simultaneously, and compatibility with additional clean-up and sample preparation methods presented by LC-MS/MS make this a promising analytical tool for 8-oxoGua determination and/or the overall biomarkers of DNA[8, 9, 15], however it requires skilled personnel and its cost make this approach economically unfeasible for routine implementation.

Therefore, there is a need for simple and cost-effective assays capable of measuring DNA oxidation and electrochemical methods are very promising tools to perform these approaches[16-18], because they are easily applied and also provide rapid and accurate results, but still require improvements in terms of repeatability and reproducibility.[19] The recent progress in the development of electrochemical (bio)sensors and application in detecting oxidative DNA damages has been reviewed by Fojta et al [20]. Modification of the electrodes with biochemical transducers, chemical mediators, nanoparticles or nanomaterials, among other strategies, in order to improve the sensitivity of carbon-based electrodes is overviewed. Also, advances in the development of sensitive photoelectrochemical biosensor for monitoring the DNA biorecognition has been reviewed by Zhao et al [21]. Nevertheless, screen-printing technology, which emerged in the last decade as a revolutionary new concept of simplifying electrochemical laboratory based protocols, opening doors to unlimited designs and encouraging new applications, is becoming a very attractive alternative for the development of reliable and simple methodologies for the direct application in this field. Screen-printed electrodes have scales of economy and provide one-shot disposable and reproducible electrochemical sensors that require no pre-treatment or electrode polishing between measurements as can be the case for traditional solid electrodes.

Due to high sensitivity, voltammetric techniques have been demonstrated their suitability to detect low concentrations of 8 -oxoGua $[22,23]$. In this particular case, a minimum concentration value that 8-oxoGua is found in biological samples is challenging to define because it depends on numerous factors (intensity of induced damage among many others). Using electrochemical detection coupled to liquid chromatography, a concentration of 8-oxoGua below $100 \mathrm{nM}$ was reported as basal value excreted into urine due to aerobic cellular metabolism[15]. Other few publications reported that levels also below $100 \mathrm{nM}$ were quantified in urine samples[13, 24], with the exception or another one that presented a concentration around $580 \mathrm{nM}[25]$.

Isolated electroanalytical detection of 8-oxoGua (not coupled to other separation analytical technique) is surprisingly seldom explored in the literature. The direct electrochemical determination of 8-oxoGua was reported for the first time by Brett et al [23] utilising glassy 
117 carbon electrodes. Rebelo et al [22] also investigated the electrochemical behaviour of this 118 compound in the presence of uric acid using glassy carbon electrodes studying exhaustively the 119 influence of $\mathrm{pH}$ in the simultaneous determination. Ferantopova [26] also compared the redox transformations of 8-oxoGua, specifically as intermediate species, to study the influence of electrode material in the oxidation process of guanine using polycrystalline gold electrodes. Recently, Sanjuán et al [27] used a new three electrode configuration set-up consisting of a boron doped diamond (BDD) as working electrode and a screen-printed electrode providing only the counter and pseudo-reference electrodes for the analysis of 7-methylguanine, considering 8-oxoGua just as interference species that might compromise the analysis. Overall, the potential disadvantage is that the electrode needs extensive pre-treatment, in the form of polishing, between measurements and are expensive. In summary, a thorough study of 8-oxoGua at SPEs has not been carried out and the advantage of using these as electrochemical sensing platforms has yet to be investigated.

To the best of our knowledge, this paper is the first report of the electrochemical behaviour of 8-oxoguanine at screen-printed graphite macroelectrodes (SPEs). Differential pulse voltammetry (DPV) has been used herein to develop an electroanalytical methodology which has been successfully validated and applied for the determination of 8-oxoguanine in simulated biological samples (human semen).

\section{Experimental}

\subsection{Chemicals and solutions preparation}

All analytical and highest analytical grade chemicals were used as received without any further purification. 8-hydroxyguanine (8-oxoguanine) was received in individual $10 \mathrm{mg}$ amber glass vials purchased from Enzo Life Sciences (Exeter, UK) and kept in the freezer until used. After accurately weighted desirable amount of product for the preparation of solutions, nitrogen was gently applied into the vial to eliminate oxygen and avoid premature oxidation of 8-oxoguanine.

$1430.1 \mathrm{M}$ potassium phosphate monobasic and $0.1 \mathrm{M}$ potassium phosphate dibasic trihydrate were used to prepare phosphate buffer solutions (PBS) at different pH throughout this work. All solutions were prepared with deionised water of resistivity not less than $18.2 \mathrm{M} \Omega \mathrm{cm}\left(25^{\circ} \mathrm{C}\right)$.

147 to ensure total solubility of the compound, $20 \mu \mathrm{L}$ of $8 \mathrm{M} \mathrm{NaOH}$ solution were added to give a 148 final $\mathrm{pH}$ value of 9-10, as verified with $\mathrm{pH}$-indicator paper.[14] The solution was protected with 
aluminium foil and constantly stirred for $24 \mathrm{~h}$ and kept in the fridge until analysed. The same methodology was applied for the preparation of adenine $(A)$ and guanine $(G)$ solutions also tested in this work. The final concentration of freshly prepared 8-oxoGua, A and G solutions were determined via UV-vis spectrophotometry using an extinction coefficient of $7,762 \mathrm{~cm}^{-1} \mathrm{M}^{-}$ ${ }^{1}$ at $285 \mathrm{~nm}$ for 8-oxoGua[28], an extinction coefficient of $13,400 \mathrm{~cm}^{-1} \mathrm{M}^{-1}$ at $261 \mathrm{~nm}$ for adenine[29] and an extinction coefficient of $10,700 \mathrm{~cm}^{-1} \mathrm{M}^{-1}$ at $243 \mathrm{~nm}$ for guanine.[30]

\subsection{Fabrication of screen-printed graphite macroelectrodes (SPES)}

Screen-printed graphite macrcoelectrodes (SPEs) which have $3 \mathrm{~mm}$ diameter working electrode, were fabricated in-house with appropriate stencil designs using a DEK 248 screen-printing machine (DEK, Weymouth, UK). A previously used carbon-graphite ink formulation (product code: C2000802P2; Gwent Electronic Materials Ltd, UK) was first screen-printed onto a polyester flexible film (Autostat, $250 \mu \mathrm{m}$ thickness). This layer was cured in a fan oven at $60{ }^{\circ} \mathrm{C}$ for 30 minutes. Next a silver/silver chloride pseudo reference electrode was included by screenprinting $\mathrm{Ag} / \mathrm{AgCl}$ paste (product code: C2030812P3; Gwent Electronic Materials Ltd, UK) onto the polyester substrate. A dielectric paste/ink (product code: D2070423D5; Gwent Electronic Materials Ltd, UK) was next printed to cover the connections. After curing again at the same conditions as before, the screen-printed electrodes were ready to be used. The SPEs were then precisely cut to remove the $\mathrm{Ag} / \mathrm{AgCl}$ pseudo reference and carbon counter and used into a standard three electrodes configuration.

\subsection{Electrochemical measurements}

Electrochemical measurements were carried out with a Palmsens (Palm Instruments BV, The Netherlands) potentiostat controlled by software PSTrace 4.7. All experiments throughout this study were conducted using a three electrodes configuration electrochemical cell with SPEs, platinum wire and saturated calomel electrode (SCE) as a working, counter and reference electrodes, respectively. All electrochemical experiments were performed at room temperature and solutions were not deaerated before analysis.

Screen-printed graphite macroelectrodes were electrochemically conditioned before use by applying 10 scans in cyclic voltammetry (CV) between $0.0 \mathrm{~V}$ and $+1.0 \mathrm{~V}$ at $50 \mathrm{mV} \mathrm{s}^{-1}$ in PBS pH 7.44. Instrumental parameters of differential pulse voltammetry (DPV) method were thoroughly optimised based on current intensity of the oxidation of 8-oxoGua and final conditions used were: step potential $5 \mathrm{mV}$, pulse amplitude $90 \mathrm{mV}$, pulse width $200 \mathrm{~ms}$ and scan rate $5 \mathrm{mV} \mathrm{s}^{-1}$. 
180 CV method was also separately performed for scan rate study of 8-oxoGua at SPEs using 5, 10, $18115,20,30,40$ and $60 \mathrm{mV} \mathrm{s}^{-1}$.

182

183

\subsection{Human semen simulant preparation}

Voltammetric methodology developed for the detection of 8-oxoGua was applied in synthetic human semen simulant prepared in the laboratory. Following the protocol proposed by Owen and Katz (2005)[31], formulation (final volume $100 \mathrm{~mL}$ ) consisted of: sodium phosphate monobasic monohydrate $(5.46 \mathrm{~mL}$ of $0.123 \mathrm{M})$, sodium phosphate dibasic anhydrate $(49.14 \mathrm{~mL}$ of $0.123 \mathrm{M})$, sodium citrate dehydrate $(813 \mathrm{mg})$, potassium chloride $(90.8 \mathrm{mg})$, potassium hydroxide (88.1 mg), fructose (272 mg), glucose anhydrous (102 mg), lactic acid (62 mg), urea (45 mg), bovine serum albumin (5.04 g), calcium chloride dihydrate (101 mg dissolved in 15.13 $\mathrm{mL}$ of water), magnesium chloride hexahydrate (92 mg dissolved in $15.13 \mathrm{~mL}$ of water) and zinc chloride (34.4 mg dissolved in $15.13 \mathrm{~mL}$ of water). In the final solution, the $\mathrm{pH}$ was raised to 7.76 adding small amounts of sodium hydroxide, then filtered with $0.45 \mu \mathrm{m}$ PTFE filter disc and kept in the fridge until assayed.

\section{Results and discussion}

\subsection{Electrochemical response of 8-oxoGua at screen-printed macroelectrodes (SPEs)}

We first explored the voltammetric behaviour of 8-oxoGua at screen-printed graphite macroelectrodes (SPEs). Cyclic voltammetry (CV) in PBS pH 7.44 was first applied for the electrochemical pretreatment of the surface of SPEs after which the differential pulse voltammetric (DPV) response of 8-oxoGua in PBS 7.57 was explored. A very well-defined and intense anodic peak at $245 \mathrm{mV}$ was observed, as depicted within Figure 1. Although it is reported that the oxidation of 8-oxoGua by ROS can generate a complex mixture of products which can be even electrochemically detected suggesting different electrode processes involved[23], we have focused our studies on the main peak observed and its voltammetric behaviour in different $\mathrm{pH}$ conditions. Note that it will be frequently observed throughout this work a small oxidation peak prior to the electrochemical 8-oxoGua signal (around -100 or -200 mV vs. SCE, depending on $\mathrm{pH}$ ) probably related to the oxidation of any species on the surface of the electrode but which is not influencing the detection of the target compound.

To further explore the electrochemical response of 8-oxoGua at SPEs, a voltammetric scan rate study was carried out by applying $\mathrm{CV}$ in the presence of $6 \mu \mathrm{M}$ of 8-oxoGua in PBS pH 7.07 at 5, 
211 10, 15, 20, 30, 40 and $60 \mathrm{mV} \mathrm{s}^{-1}$ scan rates (Figure ESI 1A). It is clearly observed a linear

212 dependency of peak current intensity $\left(I_{p}\right) v s$. square root of the scan rate $\left(v^{1 / 2}\right)$ and peak current

$213 \operatorname{logarithm}\left(\log I_{p}\right)$ vs. scan rate logarithm (Log v) (Figures ESI 1B and 1C). The analysis of this data

214 indicates that the oxidation of 8-oxoGua at SPEs is diffusion controlled which is also confirmed

215 by the gradient of the linear plot of $\log I_{p} v s . \log v\left(\log I_{p}=0.5046 \log v+0.1233 ; R^{2}=0.9872\right)$

216 close to the theoretical value of 0.5 . This observation is in agreement with previous results

217 reported at a glassy carbon electrode[23]. Moreover, the observed linear dependency of peak

218 potential with scan rate[27] (Figure ESI 1D) denotes irreversibility of the electrode process of 8-

219 oxoGua that is not completely in agreement with a previous study performed using a glassy

220 carbon electrode[23] where authors reported the existence of a reverse peak of 8-oxoGua in

221 square wave voltammetry (SWV), but of course we are comparing two different electrode

222 surfaces/materials; however, non-observation of reduction peak in cyclic voltammetry could be

223 due to very fast hydrolysis of 8-oxoGua.[32]

224 We next investigated the effect of $\mathrm{pH}$ upon the electrochemical oxidation of 8-oxoGua at SPEs.

225 Considering that normal $\mathrm{pH}$ values of different biological samples in real conditions, $\mathrm{pH}-$

226 dependence study over the range of 4.00 to 8.00 was systematically performed using different

227 phosphate buffer solutions. Different SPEs were conditioned and used for each pH assayed. As

228 shown in Figure 2, DPV signals of $2 \mu \mathrm{M}$ of 8-oxoGua undergo a peak potential shifting to lower

229 positive potentials from increasing the solution $\mathrm{pH}$. A linear dependence of the anodic peak of

230 8-oxoGua with $\mathrm{pH}$ is clearly observed with a slope of $61 \mathrm{mV} \mathrm{pH}^{-1}$ that is very close to the

231 theoretical Nernstian value of $59 \mathrm{mV} \mathrm{pH}^{-1}$. This suggests that, as expected and reported

232 previously on glassy carbon electrode[23], the electrochemical reaction that takes place on the

233 surface of SPE involving the same number of electrons and protons, specifically two of them in

234 the electrochemical oxidation of 8-oxoGua. On the other hand, slight changes in the peak

235 current of $2 \mu \mathrm{M}$ of 8-oxoGua at different pH studied were observed; nevertheless, a negligible

236 loss of $30 \mathrm{nA}$ of current intensity of anodic peak of 8-oxoGua at pH 8.00 compared to $\mathrm{pH} 4.00$

237 was detected. Note that it is an important advantage to consider for further applications of this

238 methodology in real conditions that possible changes in the usual $\mathrm{pH}$ of biological samples are

239 not going to compromise the voltammetric determination of 8-oxoGua in terms of peak current

240 intensity; this data suggest that SPEs are a suitable and feasible analytical approach for the in-

241 situ analysis of 8-oxoGua. 
245 The electroanalytical performance of SPEs towards the detection of 8-oxoGua was next evaluated. A calibration curve was constructed using DPV and a single SPE over the entire concentration range $(0.10$ to $12.0 \mu \mathrm{M})$ in $0.1 \mathrm{M}$ phosphate buffer solution $\mathrm{pH} 7.00$. Three replicates of each concentration were performed. Experimental observations determined that it was not necessary to clean the SPE surface between measurements. Consequently, any conditioning potential was applied before each voltammetric response apart from the initial pretreatment of the surface by applying 10 scans in $\mathrm{CV}$ at $\mathrm{pH}$ 7.44. As shown in Figure 3, there was an expected increase of the peak current upon the increasing of the concentration of 8oxoGua. A straight line calibration plot was obtained $\left(\mathrm{I} / \mu \mathrm{A}=0.0353 \mu \mathrm{A}\left[\mu \mathrm{M}^{-1}\right]+0.0049 \mu \mathrm{A} ; \mathrm{R}^{2}=\right.$ $0.9947 ; \mathrm{N}=7$ ) for the detection of 8-oxoGua over the analytical range studied (Figure 3 inset) with a limit of detection $(3 \sigma)$ found to correspond to $0.33 \mu \mathrm{M}$. In comparison to the limit of detection of $0.80 \mu \mathrm{M}$ previously reported for the direct electrochemical determination of 8oxoGua using a glassy carbon electrode[23], a significant improvement was achieved with the proposed methodology. The improved electrochemical performance over that of a GC is due to the greater density of edge plane like- sites/defects on the SPE. [33, 34]

In order to demonstrate the suitability and reliability of SPEs platforms for the determination of 8-oxoGua at different concentration levels likely to be found in real samples, the reproducibility and repeatability of 8-oxoGua voltammetric measurements at SPEs were additionally assessed at low $(1 \mu \mathrm{M})$ and high $(9 \mu \mathrm{M})$ concentrations, respectively. DPV analyses were performed in $0.1 \mathrm{M}$ phosphate buffer solution $\mathrm{pH}$ 7.00. Five different SPEs were selected for reproducibility studies and five DPV measurements were recorded for each one. Also, a single SPE was used for repeatability study and thirty times DPV measurements were continuously run using the same electrode. Note that thirty times measurements using one single SPE were considered enough to complete the study but even so the electrode did not become deteriorated. Results obtained are summarised in Table 1. In general, SPEs showed adequate relative standard deviation (RSD) values in all cases. Only the value of $11.9 \%$ for $1 \mu \mathrm{M}$ of 8-oxoGua seems to be high but still acceptable considering the low concentration analysed. Likewise, a relatively high value of $8 \%$ for repeatability of SPEs at high concentration of 8oxoGua could be related to a partial blocking of the surface because of the adsorption of the compound onto the surface after 30 runs without the application of any conditioning potential between measurements. Therefore, we have proved the feasibility of the developed methodology to perform accurately in-situ calibration and direct measurements of 8-oxoGua in practical applications using the same screen-printed electrode sensor. 
279

280

281

282

283

284

285

286

287

288

289

290

291

292

293

294

295

296

297

298

299

300

301

302

303

304

305

306

307

308

309

310

Attention was next turned to exploring the voltammetric response of 8-oxoGua simultaneously with other nucleobases in DNA, which are likely to be present within real biological samples and can be potential interfering molecules, mainly guanine $(G)$ and adenine $(A)$. A mixture of $5.6 \mu \mathrm{M}$ of 8-oxoGua, 6.3 $\mu \mathrm{M}$ of $\mathrm{G}$ and 8.8 $\mu \mathrm{M}$ of $\mathrm{A}$ in $0.1 \mathrm{M}$ phosphate buffer solution $\mathrm{pH} 7.74$ was tested using DPV and SPEs. As represented in Figure 4A, voltammetric peaks of 8-oxoGua, $G$ and $A$ appear one after the other at peak potentials about $245 \mathrm{mV}, 635 \mathrm{mV}$ and $930 \mathrm{mV}$ (vs. SCE), respectively. There is a difference of $380 \mathrm{mV}$ (vs. SCE) between 8-oxoGua and G peak potentials and $300 \mathrm{mV}$ (vs. SCE) between $\mathrm{G}$ and A peak potentials, which indicates sufficient voltammetric separation to detect them simultaneously at SPEs. Even though 8-oxoGua peak is slightly shifted in the presence of $G$ and $A$, it can be determined that there is no cooperative effect upon the voltammetric responses of each compound.[27] On the other hand, the presence of $G$ and $A$ in solution resulted in a reduction of $33 \%(46 \mathrm{nA})$ of 8-oxoGua current intensity, thus the presence of those nucleobases somewhat interferes in the electrooxidation of 8-oxoGua at SPEs. Even though SPEs did not show a clear fouling of the surface when these three compounds are simultaneously detected, as described by Sanjuán et al. (2016)[27] using BDDE, the presence of $\mathrm{G}$ and $\mathrm{A}$ are somehow competing by the adsorption sites of the SPE surface resulting in a partial blocking and decreasing of 8-oxoGua voltammetric signal.

The influence in the electrochemical response of 8-oxoGua due to other co-existent compounds with important implications in several biological processes and possibly found in real samples, was also investigated. Therefore, dopamine and ascorbic acid were also determined simultaneously with 8-oxoGua by applying the optimised DPV methodology. First, a solution

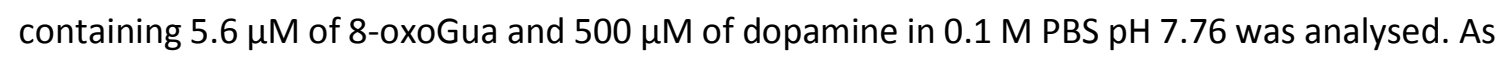
illustrated in Figure 4B, it is possible to perform their simultaneous determination since peak potentials of individual compounds are separated by $170 \mathrm{mV}$ (vs. SCE). The presence of dopamine does not affect the peak potential of 8-oxoGua, which remains unaltered, and a small reduction of $20 \%(28 \mathrm{nA}$ ) in the peak current is observed; however, a significant decrease of peak current is undertaken by dopamine with a loss of $84 \%$ of current intensity compared to voltammetric signal of the individual compound. This can be attributed to the fact that 8-oxoGua is more easily oxidised than dopamine and dominates the achievement of more active sites on the surface of SPEs blocking significantly the electrooxidation of dopamine.

On the other hand, DPV determination of $5.6 \mu \mathrm{M}$ of 8-oxoGua was additionally carried out in the presence of $27 \mu \mathrm{M}$ of ascorbic acid in $0.1 \mathrm{M} \mathrm{PBS}$ pH 7.84. Partially overlapped voltammetric 
311 peaks are observed (Figure 4C). Hence, the simultaneous detection of ascorbic acid and 8-

312 oxoGua is evidently possible but improvements in the voltammetric method and/or even

313 optimisation of $\mathrm{pH}$ conditions would be required to achieve better separation and to evaluate 314 appropriately this interference.

\subsection{Determination of 8-oxoGua in simulated human semen sample as a proof-of-concept}

The feasibility of the voltammetric detection of 8-oxoGua using SPEs was verified by applying the methodology in a human semen simulant prepared in the laboratory following the procedure described in the Experimental Section.

The complex mixture of chemicals that is part of the synthetic human semen simulation (final $\mathrm{pH}$ 7.76) provided a defined electrochemical signal at a potential of $575 \mathrm{mV}$ (vs. SCE) at SPEs using DPV; typical voltammograms are shown in Figure 5A and 5B. Experiments were performed in non-diluted and diluted 1:10 semen simulant preparations and as expected a higher current intensity was obtained for the non-diluted sample. Addition of an appropriate amount of 8oxoGua for a final concentration of $12 \mu \mathrm{M}$ within semen simulant solutions resulted in a clear voltammetric peak at $300 \mathrm{mV}$ (vs. SCE). The 8-oxoGua signal was significantly higher in diluted simulated semen sample, as expected, but in both cases, the DPV response was well-defined and sufficient intense for certainly quantification. Due to the complex mixture of chemicals that comprise the semen simulant a shift in the of 8-oxoGua signal / peak to more positive potentials, compared to the response / peak determined in simply phosphate buffer solution at the same $\mathrm{pH}$ is evident. Even though the current intensity of voltammetric peak of 8-oxoGua showed a significant reduction (about 50\%) when analysed in synthetic biological solutions, the reliability of SPEs for its detection and appropriately quantification in practical conditions has been demonstrated. 
342 This paper has investigated, for the first time, the utilisation of screen-printed graphite 343 electrodes for the potential evaluation of DNA damage via the electrochemical determination 344 of 8-oxoguanine. CV and DPV were carefully optimised for high-performance of screen-printed 345 platforms for the direct quantification of 8-oxoGua.

346 According to insignificant changes in the current intensity of the target compound over the 347 range of $\mathrm{pH}$ studied (4.00 to 8.00 ), the developed methodology demonstrated its suitability for 348 8-oxoGua determination at usual $\mathrm{pH}$ of biological samples. The proposed methodology was also 349 validated in terms of linearity obtaining a good linear regression in the concentration range of $350 \quad 0.1-12 \mu \mathrm{M}$ with a sensitivity of $0.0353 \mu \mathrm{A} \mu \mathrm{M}^{-1}$. A very good LOD of $0.33 \mu \mathrm{M}$ was obtained and 351 it was significantly better than LOD reported for conventional glassy carbon electrodes $(0.80$ $352 \mu \mathrm{M})[23$ ]. Additionally, reproducibility of different screen-printed platforms and repeatability on 353 a single sensor were performed at high and low concentrations and they provided acceptable

354 RSD values demonstrating the reliability of SPEs for direct determination of 8-oxoGua.

355 Simultaneous detection of 8-oxoGua in the presence of other important nucleobases (adenine 356 and guanine) and some co-existent compounds in biological fluids were also successfully carried 357 out. Finally, the successful application of the developed methodology for the determination of 358 8-oxoGua in synthetic human semen demonstrating the feasibility of SPEs for further biomedical applications; such work is underway.

Acknowledgements 
369 Table 1. Reproducibility and repeatability of $1 \mu \mathrm{M}$ and $9 \mu \mathrm{M}$ DPV responses of 8-oxoGua at SPEs 370 in $0.1 \mathrm{M} \mathrm{PBS}$ pH 7.00. $\mathrm{N}$ value for reproducibility corresponds to different electrodes used while 371 the $\mathrm{N}$ value for repeatability indicates the number of times of repetition of voltammetric 372 measurements using a single SPE.

\begin{tabular}{|l|c|c|}
\cline { 2 - 3 } \multicolumn{1}{c|}{} & \multicolumn{2}{c|}{ Concentration of 8-oxoGua } \\
\cline { 2 - 3 } \multicolumn{1}{c|}{} & $\mathbf{1} \boldsymbol{M}$ & $\mathbf{9} \mu \mathrm{M}$ \\
\hline Reproducibility (N=5) & $0.028 \pm 0.003 \mu \mathrm{A}$ (RSD 11.9\%) & $0.298 \pm 0.013 \mu \mathrm{A}$ (RSD 4.5 \%) \\
\hline Repeatability (N=30) & $0.023 \pm 0.001 \mu \mathrm{A}(\mathrm{RSD} 6.2 \%)$ & $0.319 \pm 0.026 \mu \mathrm{A}$ (RSD 8.0 \%) \\
\hline
\end{tabular}

373

374

375 
376 Figure 1. Differential pulse voltammetry of $20 \mu \mathrm{M}$ of 8-oxoGua in $0.1 \mathrm{M} \mathrm{PBS} \mathrm{pH} 7.57$ (solid line) 377 using SPEs. The background (no 8-oxoguanine) was recorded and is presented as the dashed 378 line.

379

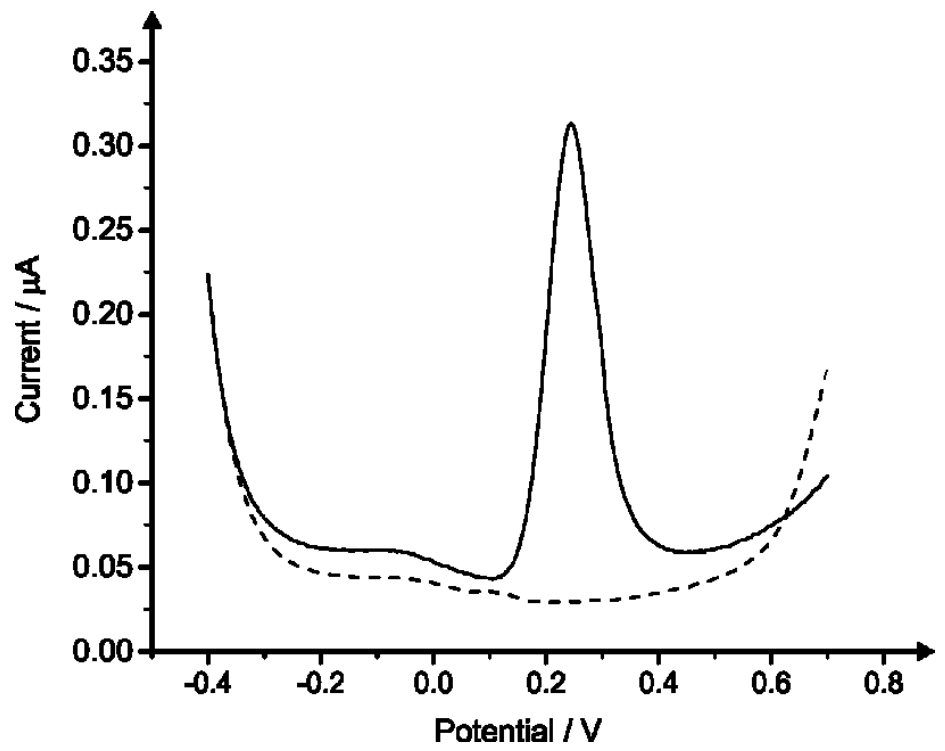

381 
382 Figure 2. Effect of $\mathrm{pH}$ on the DPV responses of $2 \mu \mathrm{M}$ of 8-oxoGua in $0.1 \mathrm{M}$ PBS using SPEs. Inset 383 Figure depicts the decreasing of peak potential of 8-oxoGua with changing the solution $\mathrm{pH}$.

384 
Figure 3. DPV measurements of standard solutions of 8-oxoGua $(0.1,0.3,1.0,3.0,6.0,9.0$ and $12.0 \mu \mathrm{M}$ ) in 0.1M PBS pH 7.44 using a single SPE. Figure inset shows linear a calibration plot of current intensity vs. concentration of 8-oxoGua. The insert plot shows an average and standard deviation at each concentration.

392

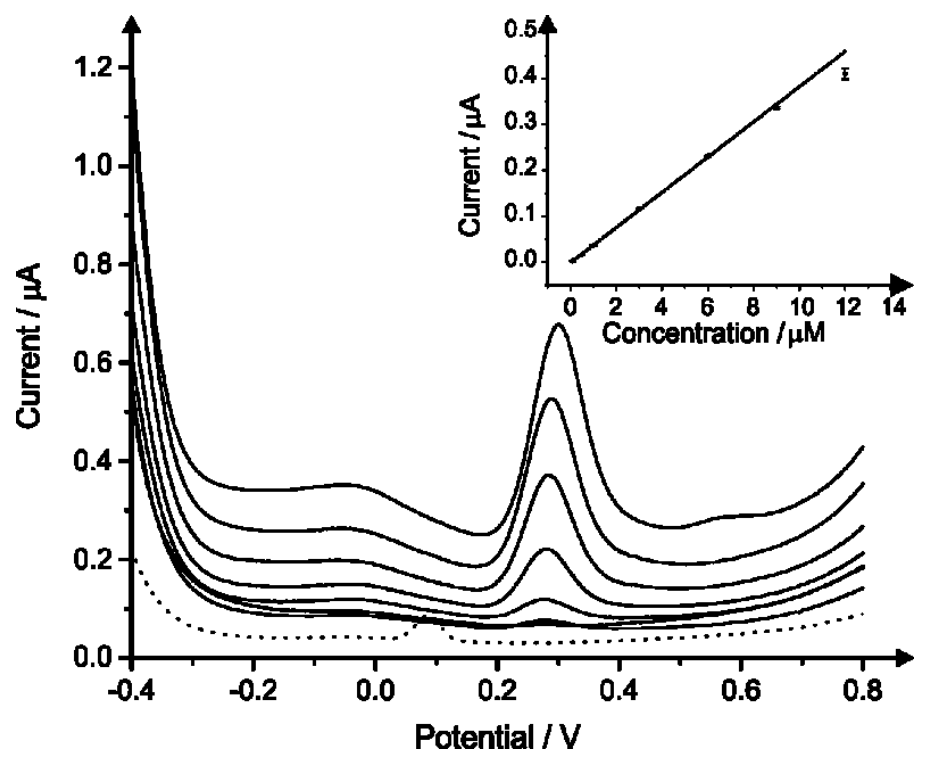

393

Potential /V 
400
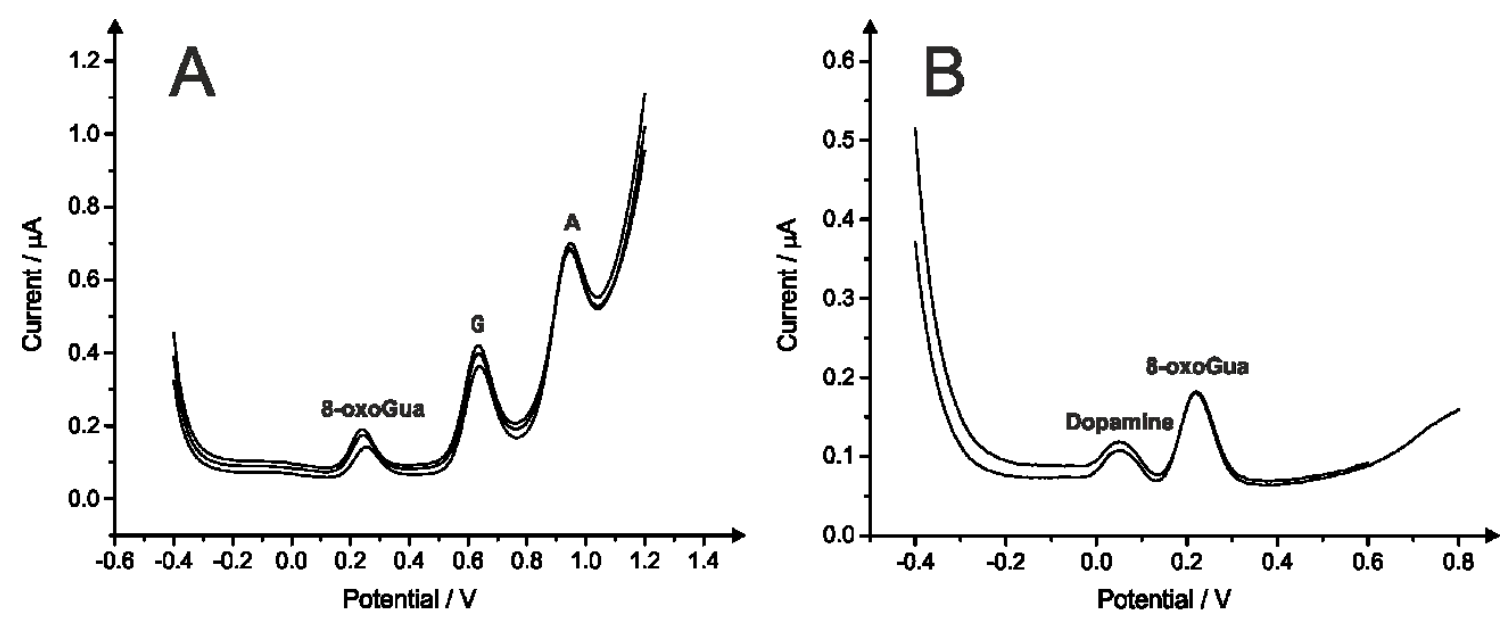

Figure 4. DPV consecutive measurements of 8-oxoGua in the presence of guanine and adenine in $0.1 \mathrm{M}$ PBS pH 7.74 (A). DPV curves of the simultaneous determination of 8-oxoGua and dopamine in $0.1 \mathrm{M} \mathrm{PBS}$ pH 7.76 (B). DPV responses of a mixture of 8-oxoGua and ascorbic acid in $0.1 \mathrm{M} \mathrm{PBS}$ pH 7.84 (C).

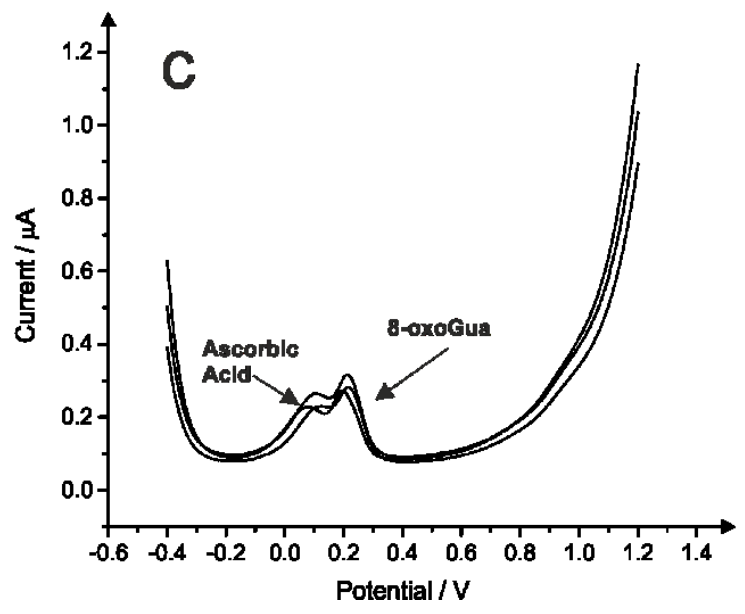


Figure 5. Voltammetric responses of 8-oxoGua in a complex human semen simulant preparation

405

406
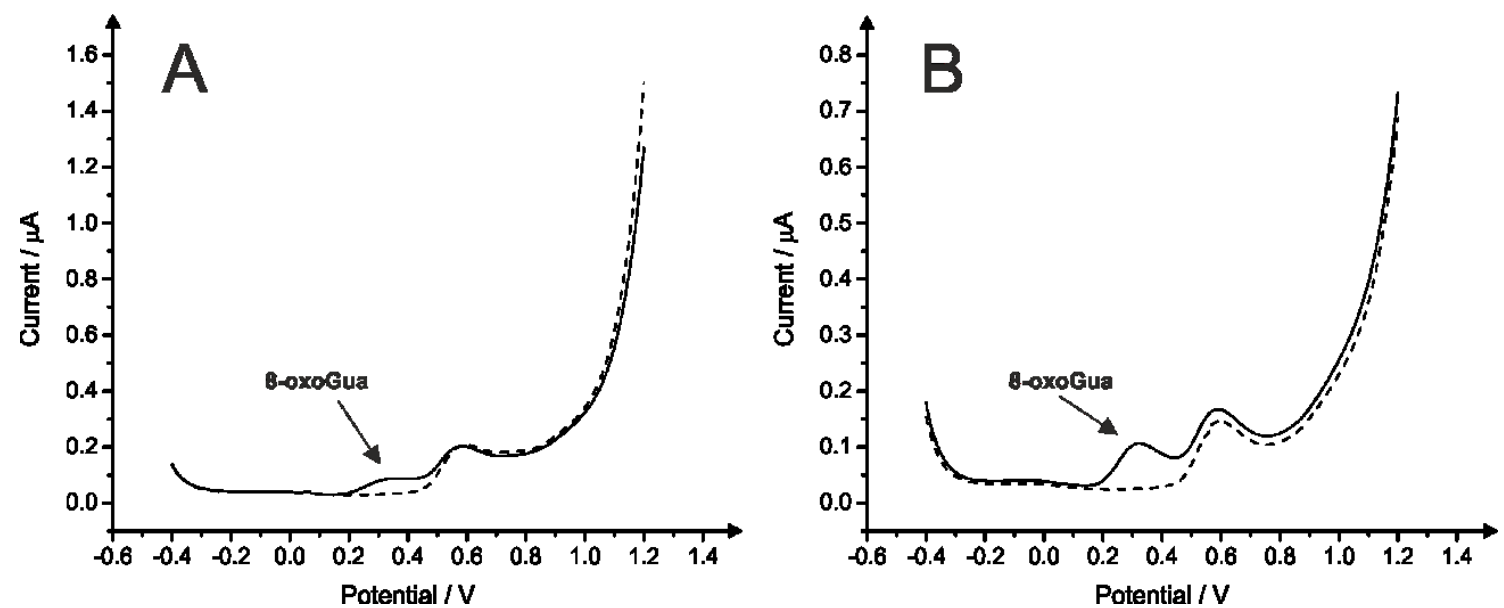

407 
409 Figure ESI 1. CV responses of $6 \mu \mathrm{M}$ 8-oxoGua in $0.1 \mathrm{M} \mathrm{PBS} \mathrm{pH} 7.07$ as a function of scan rate (A) 410 at SPEs. Plots of peak current $\left(I_{p}\right) v s$. square root of the scan rate $\left(v^{1 / 2}\right)(B)$, peak current logarithm $411\left(\log I_{p}\right)$ vs. scan rate logarithm $(\log v)(C)$ and peak potential $\left(E_{p}\right)$ vs. scan rate $(v)$.

412
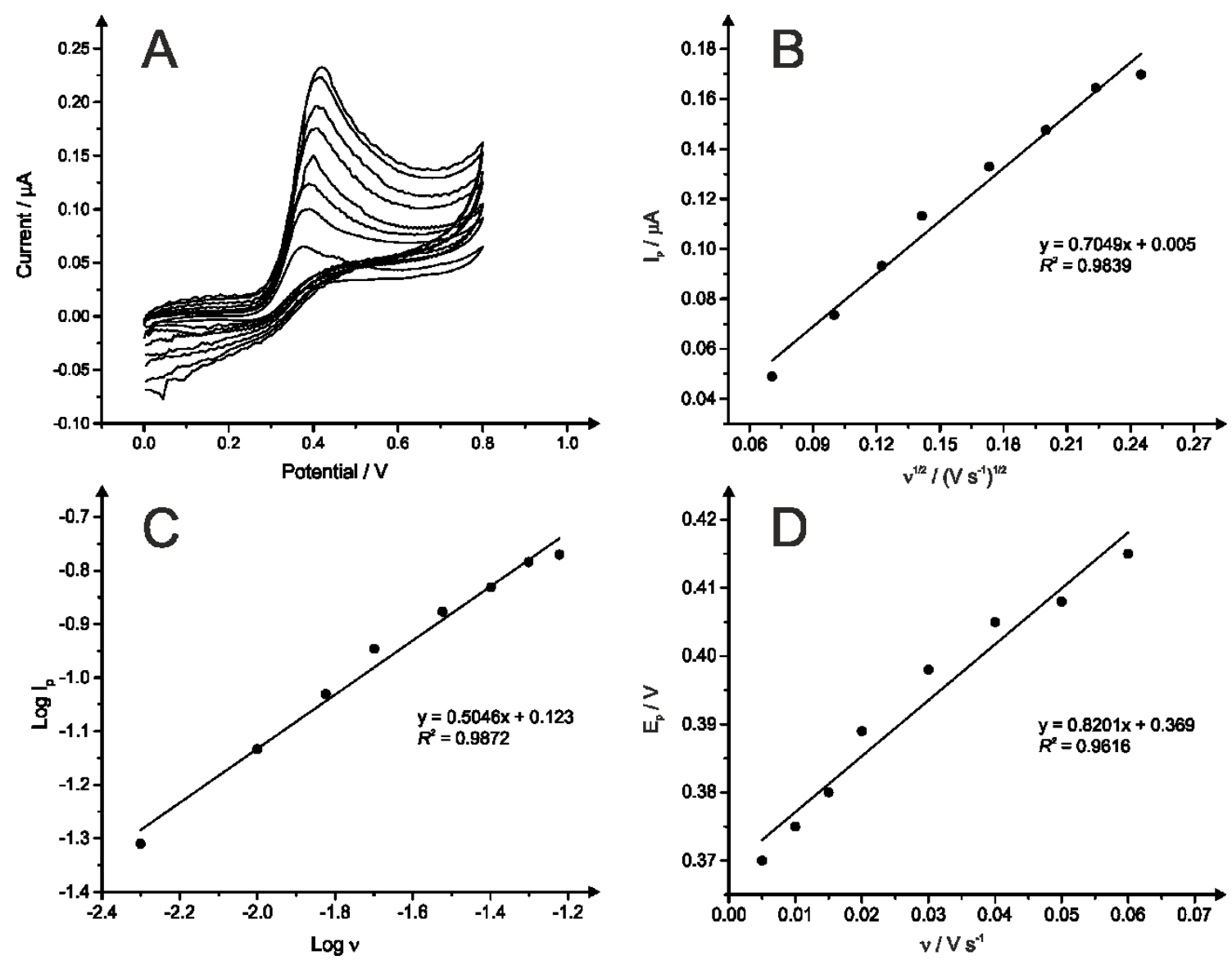
417 [1] M.W. Germann, C.N. Johnson, A.M. Spring, Recognition of Damaged DNA: Structure and 418 Dynamic Markers, Medicinal Research Reviews, 32(2012) 659-83.

419 [2] Y.-H. Su, Y.-L. Lee, S.-F. Chen, Y.-P. Lee, Y.-H. Hsieh, J.-H. Tsai, et al., Essential role of $\beta$-human 420 8-oxoguanine DNA glycosylase 1 in mitochondrial oxidative DNA repair, Environmental and 421 Molecular Mutagenesis, 54(2013) 54-64.

422 [3] N. Kumar, P.K. Shukla, P.C. Mishra, Reactions of the $\mathrm{OOH}$ radical with guanine: Mechanisms of formation of 8-oxoguanine and other products, Chemical Physics, 375(2010) 118-29.

[4] R. Colnaghi, G. Carpenter, M. Volker, M. O'Driscoll, The consequences of structural genomic alterations in humans: Genomic Disorders, genomic instability and cancer, Seminars in Cell \& Developmental Biology, 22(2011) 875-85.

[5] A. Bacsi, L. Aguilera-Aguirre, B. Szczesny, Z. Radak, T.K. Hazra, S. Sur, et al., Down-regulation of 8-oxoguanine DNA glycosylase 1 expression in the airway epithelium ameliorates allergic lung inflammation, DNA Repair, 12(2013) 18-26.

[6] S. Steenken, S.V. Jovanovic, How Easily Oxidizable Is DNA? One-Electron Reduction Potentials of Adenosine and Guanosine Radicals in Aqueous Solution, Journal of the American Chemical Society, 119(1997) 617-8.

[7] P. German, D. Saenz, P. Szaniszlo, L. Aguilera-Aguirre, L. Pan, M.L. Hegde, et al., 8-Oxoguanine DNA glycosylase1-driven DNA repair-A paradoxical role in lung aging, Mechanisms of Ageing and Development, 161(2017) 51-65.

[8] W.M. Winnik, K.T. Kitchin, Measurement of oxidative stress parameters using liquid chromatography-tandem mass spectroscopy (LC-MS/MS), Toxicology and Applied Pharmacology, 233(2008) 100-6.

[9] B. Malayappan, T.J. Garrett, M. Segal, C. Leeuwenburgh, Urinary analysis of 8-oxoguanine, 8oxoguanosine, fapy-guanine and 8-oxo-2'-deoxyguanosine by high-performance liquid chromatography-electrospray tandem mass spectrometry as a measure of oxidative stress, Journal of Chromatography A, 1167(2007) 54-62.

[10] J.-L. Ravanat, E. Gremaud, J. Markovic, R.J. Turesky, Detection of 8-Oxoguanine in Cellular DNA Using 2,6-Diamino-8-oxopurine as an Internal Standard for High-Performance Liquid Chromatography with Electrochemical Detection, Analytical Biochemistry, 260(1998) 30-7.

[11] J.-L. Ravanat, R.J. Turesky, E. Gremaud, L.J. Trudel, R.H. Stadler, Determination of 8Oxoguanine in DNA by Gas Chromatography-Mass Spectrometry and HPLC-Electrochemical Detection: Overestimation of the Background Level of the Oxidized Base by the Gas Chromatography-Mass Spectrometry Assay, Chemical Research in Toxicology, 8(1995) 1039-45. [12] K.E. Herbert, M.D. Evans, M.T.V. Finnegan, S. Farooq, N. Mistry, I.D. Podmore, et al., A novel HPLC procedure for the analysis of 8-oxoguanine in DNA, Free Radical Biology and Medicine, 20(1996) 467-73.

[13] L. Long, D.R. McCabe, M.E. Dolan, Determination of 8-oxoguanine in human plasma and urine by high-performance liquid chromatography with electrochemical detection, Journal of Chromatography B: Biomedical Sciences and Applications, 731(1999) 241-9.

[14] I.A. Rebelo, J.A.P. Piedade, A.M. Oliveira-Brett, Development of an HPLC method with electrochemical detection of femtomoles of 8-oxo-7,8-dihydroguanine and 8-oxo-7,8-dihydro2'-deoxyguanosine in the presence of uric acid, Talanta, 63(2004) 323-31.

[15] A. Weimann, D. Belling, H.E. Poulsen, Quantification of 8-oxo-guanine and guanine as the nucleobase, nucleoside and deoxynucleoside forms in human urine by high-performance liquid chromatography-electrospray tandem mass spectrometry, Nucleic Acids Research, 30(2002) 18.

[16] K.C. Honeychurch, M.R. O'Donovan, J.P. Hart, Voltammetric behaviour of DNA bases at a screen-printed carbon electrode and its application to a simple and rapid voltammetric method for the determination of oxidative damage in double stranded DNA, Biosensors and Bioelectronics, 22(2007) 2057-64. 
[17] A. Brotons, L.A. Mas, J.P. Metters, C.E. Banks, J. Iniesta, Voltammetric behaviour of free DNA 468 bases, methylcytosine and oligonucleotides at disposable screen printed graphite electrode 469 platforms, Analyst, 138(2013) 5239-49.

470 [18] E.P. Randviir, C.E. Banks, Electrochemical measurement of the DNA bases adenine and 471 guanine at surfactant-free graphene modified electrodes, RSC Advances, 2(2012) 5800-5.

472 [19] A. Brotons, I. Sanjuan, C.E. Banks, F.J. Vidal-Iglesias, J. Solla-Gullón, J. Iniesta, Voltammetric 473 Behaviour of 7-Methylguanine Using Screen-printed Graphite Electrodes: towards a Guanine 474 Methylation Electrochemical Sensor, Electroanalysis, 27(2015) 2766-72.

475 [20] M. Fojta, A. Daňhel, L. Havran, V. Vyskočil, Recent progress in electrochemical sensors and assays for DNA damage and repair, TrAC Trends in Analytical Chemistry, 79(2016) 160-7.

[21] W.-W. Zhao, J.-J. Xu, H.-Y. Chen, Photoelectrochemical DNA Biosensors, Chemical Reviews, 114(2014) 7421-41.

479 [22] I. Rebelo, J.A.P. Piedade, A.M. Oliveira Brett, Electrochemical determination of 8oxoguanine in the presence of uric acid, Bioelectrochemistry, 63(2004) 267-70.

[23] A.M.O. Brett, J.A.P. Piedade, S.H.P. Serrano, Electrochemical Oxidation of 8-Oxoguanine, Electroanalysis, 12(2000) 969-73.

[24] E.M. Park, M.K. Shigenaga, P. Degan, T.S. Korn, J.W. Kitzler, C.M. Wehr, et al., Assay of excised oxidative DNA lesions: isolation of 8-oxoguanine and its nucleoside derivatives from biological fluids with a monoclonal antibody column, Proceedings of the National Academy of Sciences of the United States of America, 89(1992) 3375-9.

[25] J.-L. Ravanat, P. Guicherd, Z. Tuce, J. Cadet, Simultaneous Determination of Five Oxidative DNA Lesions in Human Urine, Chemical Research in Toxicology, 12(1999) 802-8.

[26] E.E. Ferapontova, Electrochemistry of guanine and 8-oxoguanine at gold electrodes, Electrochimica Acta, 49(2004) 1751-9.

[27] I. Sanjuán, A. Brotons, N. Hernández-Ibáñez, C.W. Foster, C.E. Banks, J. Iniesta, Boron-doped diamond electrodes explored for the electroanalytical detection of 7-methylguanine and applied for its sensing within urine samples, Electrochimica Acta, 197(2016) 167-78.

[28] D. Ampadu Boateng, Kinetics of Formation and Oxidation of 8-oxo-7,8-dihydroguanine (80xoG): East Tennessee State University; 2014.

[29] M.S. Swaraga, M. Adinarayana, Kinetics and mechanism of protection of adenine from sulphate radical anion by caffeic acid under anoxic conditions, Indian Journal of Biochemistry and Biophysics, 40(2003) 27-30.

[30] G. Fasman, C. CR, Handbook of Biochemistry and Molecular Biology: CRC, Press; 1977.

[31] D.H. Owen, D.F. Katz, A Review of the Physical and Chemical Properties of Human Semen and the Formulation of a Semen Simulant, Journal of Andrology, 26(2005) 459-69.

[32] R.N. Goyal, G. Dryhurst, Redox chemistry of guanine and 8-oxyguanine and a comparison of the peroxidase-catalyzed and electrochemical oxidation of 8-oxyguanine, Journal of Electroanalytical Chemistry and Interfacial Electrochemistry, 135(1982) 75-91.

[33] R.O. Kadara, N. Jenkinson, C.E. Banks, Characterisation of commercially available electrochemical sensing platforms, Sensors and Actuators B: Chemical, 138(2009) 556-62.

[34] P. Fanjul-Bolado, D. Hernández-Santos, P.J. Lamas-Ardisana, A. Martín-Pernía, A. CostaGarcía, Electrochemical characterization of screen-printed and conventional carbon paste electrodes, Electrochimica Acta, 53(2008) 3635-42. 